\title{
Flow Field and Temperature Field of Water-Cooling-Type Magnetic Coupling
}

\author{
Lei Wang ${ }^{1,2}$, Zhenyuan Jia', Yuqin Zhu² and Li Zhang ${ }^{2 *}$
}

\begin{abstract}
At present, the water-cooling simulation of the water-cooled magnetic coupler is based on the water-cooled motor and the hydraulic coupler, which cannot accurately characterize the temperature distribution of the rotating watercooled coupling of the coupler. Focusing on rotating water cooling radiating, the present paper proposes simulating the water cooling temperature field as well as the flow field through the method of combining fluid-solid coupled heat transfer and MRF (Multiphase Reference Frame). In addition, taking an $800 \mathrm{~kW}$ magnetic coupling as an example, the paper optimizes the shape, number, cooling water inlet speed, and so on, of the cooling channel. Considering factors such as the complete machine's temperature, and drag torque, it is proved that the cooling effect is best when there are 36 involute curved channels and when the inlet speed is $3 \mathrm{~m} / \mathrm{s}$. Further, through experiments, the actual temperature values at six different positions when $50 \mathrm{~kW}$ and $70 \mathrm{~kW}$ thermal losses differ are measured. The measured values agree with the simulation results, proving the correctness of the proposed method. Further, data have been collected during the entire experimental procedure, and the variation in the coupling's temperature is analyzed in depth, with the objective of laying a foundation for the estimation of the inner temperature rise as well as for the optimization of the structural design.
\end{abstract}

Keywords: Water-cooling magnetic coupling, Fluid-solid coupling, Channel, Three-dimensional temperature field

\section{Introduction}

Magnetic coupling is a new type of non-contact pure mechanical transmission gear [1]. It is applicable to severe environments with conditions such as high humidity and dust [2], and it can realize a flexible transmission connection from motor to load by sensing the mutual effect between the magnetic fields with high transmission efficiency [3]. Magnetic coupling provides torque mainly through a copper disk vortex, which will cause loss at the same time and become a major heat release source [4]. As a result, the equipment's temperature will increase [5]. In particular, the heating value of a high-power coupling will be considerable [6]. When the temperature is excessively high, the copper disk will become soft and lose its shape under the centrifugal effect [7]. In this way, the dynamic balancing performance will be reduced substantially,

\footnotetext{
*Correspondence: 89378317@qq.com

${ }^{2}$ State Key Laboratory of Coal Mine Safety Technology, CCTEG Shenyang Research Institute, Fushun 113122, China

Full list of author information is available at the end of the article
}

causing violent vibration, which will seriously damage the bearing [8]; at the same time, high temperature will cause the thread fastening agent to melt. Finally, the bolt will fall, leading to equipment breakdown and danger to the lives of personnel [9]. Moreover, the heat resistance of the coupling's key part, namely, the permanent magnet, is poor [10]. Excessive temperature will create irreversible demagnetization of the permanent magnet [11, 12]. Hence, there are potential safety hazards when the temperature becomes too high that negatively affect the equipment's stable operation. As a result, it is necessary to design a special water cooling system for the heat radiating from the heat source, the copper disk.

Water cooling radiating is commonly used in equipment, such as high-power motors, CPUs, and frequency converters [13]. The cooling method is static heat transfer of the cooling water jacket and heat source [14]. The cooling water can take away heat when it flows through the channel [15]. For example, a high-power motor's water cooling radiating system [16], which adopts the approach of a cooling water jacket enclosing the motor 
stator, is motor radiating, whose coolant galleries mainly have a Z-shaped circumference [17], spiral-shaped circumference [18], half-spiral-shaped circumference [19], Z-shaped axial direction [20], and so on [21]. When the cooling water jacket is static, temperature field simulation can be carried out if the relevant conditions, such as cooling water flow or speed are given [22]. The calculation process is relatively simple. The water cooling systems of the CPU and the frequency converter are similar to that of the motor [23, 24], which all employ a cooling channel and heat source static heat transfer and employ similar calculation methods as those utilized in traditional water cooling simulation.

However, the heat source part of the magnetic coupling is a high-speed rotational rotor, making it impossible to employ the calculation method used in the traditional cooling method. Water cooling magnetic coupling usually achieves the aim of heat dissipation by opening a channel on the heat source rotor and the flow of cooling water. The heat source rotor rotates at a high speed. In addition, the inlet and outlet of water are open to the outside. The cooling water also rotates at a high speed with the channel, and its flow field movement as well as distribution are extremely uncertain. When the fluid-solid coupled heat transmission process of cooling water and copper disk and steel disk is transferred to the simulation, it is not easy to establish an equivalent model, and defining the boundary conditions is complicated, which makes it difficult to carry out the coupling's water cooling thermodynamic calculation as well as the simulation calculation. There are few studies on the flow field and temperature field of high-speed revolution flow cooling. Moreover, studies concerning high-power coupling of the water cooling flow field and the temperature field are even fewer. Only Dai et al. [25] have carried out a two-dimensional flow field simulation calculation on a high-power water cooling cylinder permanent magnet governor and corrected the convection heat release coefficient on the outer surface of the conductor cylinder. However, their calculation model uses a cylinder-type magnetic governor, in which the cooling water flows inside the peripheral circular ring and does not rotate with the rotor. Because it employs the traditional water cooling radiating simulation method, it cannot represent the complicated heat transfer model of cooling water rotating with a rotor.

The present paper adopts the calculation method of the unsteady state fluid-solid coupling heat transfer model combined with multiphase reference frame (MRF). This is to carry out a thermodynamic simulation of the flow field and temperature field of an $800 \mathrm{~kW}$ water cooling magnetic coupling rotating heat source, optimize the channel, test three different curved channels of varying shapes, and determine the optimal channel shape. Meanwhile, the impact of varying the number of distinct channels and inlet speed on the temperature field under the optimal flow channel shape are also calculated, in order to optimize the flow field and the temperature field. Finally, an experimental platform of water cooling coupling is created. The above-mentioned calculation methods, as well as the optimized cooling channels, are verified through experiments that prove the correctness of the calculation methods and the soundness of the optimized results, offering reliable calculation methods for cooling water rotating fluid-solid coupling heat transfer.

\section{Configuration of the Exoskeleton Arm System}

The structure diagram of the water-cooling-type magnetic coupling is shown in Figure 1. It is mainly composed of the magnetic coupling body, the outer box, and the circulating water cooling system. The coupling body, which is composed of the input module and the output module, is shown in Figure 2. A copper disk, steel disk, and so on, comprise the input module, which is connected to the motor; a permanent magnet, aluminum disk, magnet cover, and so on, make up the outlet module, which is connected to the load end. Its working principle is as follows: the motor starts and drives the coupling's input end's rotor to rotate. When the input end rotor's copper disk rotates, relative motion is produced between the copper disk and the permanent magnet. Through the air gap, the alternating magnetic field generates a vortex on the copper disk. At the same time, the vortex produces an induced magnetic field, which interacts with the original magnetic field, making the permanent magnet rotate along the same direction as the copper disk, which obtains the transmission from the motor torque to load torque and then drives the load to rotate.

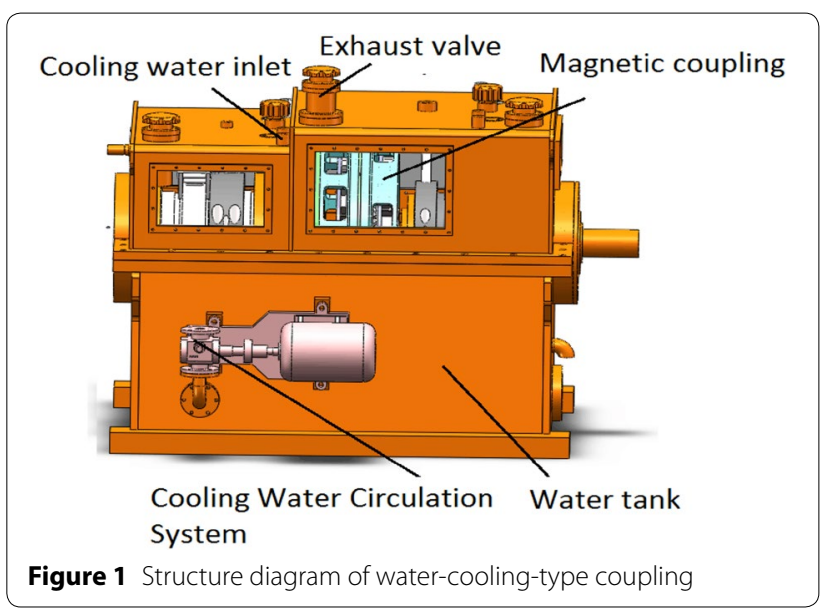




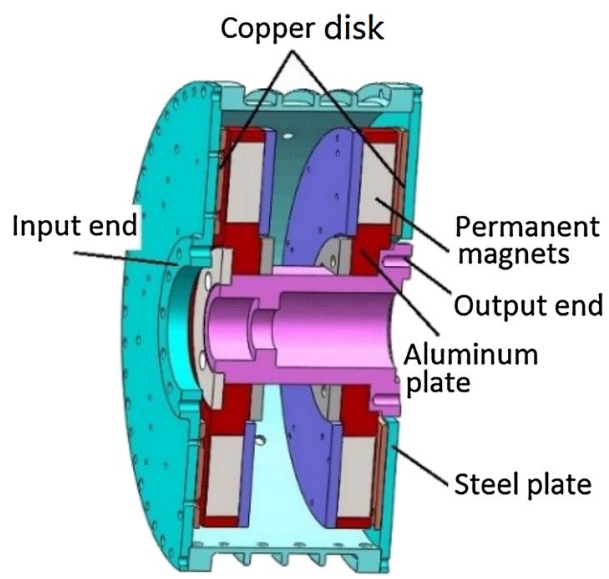

Figure 2 Structure diagram of coupling body

During the torque transmission, there will also be vortex loss, which is mainly the copper disk vortex loss, a heat source that will lead to a temperature rise in the copper disk and associated components. Due to the high temperature, the copper disk may become soft, the thread fastening agent may melt, and the permanent magnet may demagnetize. It is necessary to arrange a cooling water channel to cool the copper disk. Theoretically, it is best to open a flow channel on the copper disk to cool it directly. However, as the key part of vortex, the integrity of the copper disk is of vital importance to the magnetic field. Therefore, it is not advisable to open a flow channel on the copper disk. Alternatively, a flow channel is opened on the steel disk connected to the copper disk. In this way, not only can the integrity of the vortex field be guaranteed, but also the heat release effect can be maximized. The cooling water circulating process and the cooling flow channel of the steel disk are shown in Figure 3.
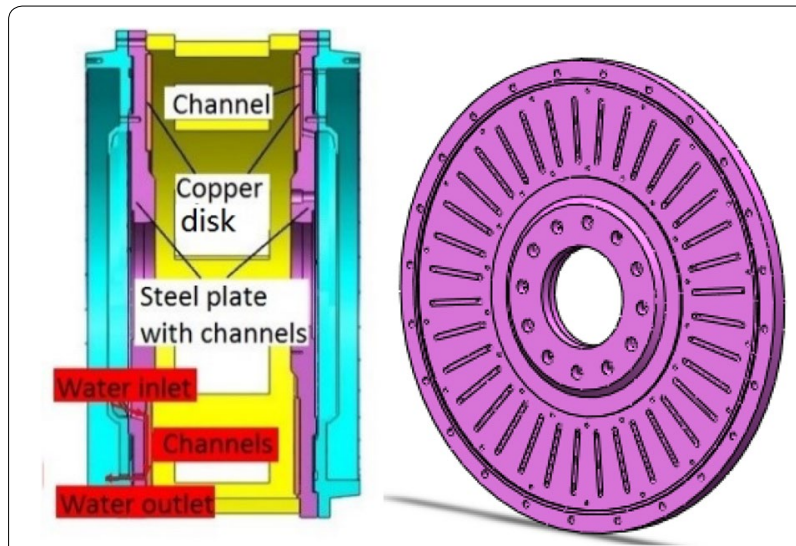

Figure 3 Cooling process and cooling channel

\section{Thermal-fluid Coupled Analysis of 3D Numerical Model}

\subsection{Physical Model and Mesh Generation}

The present paper takes an $800 \mathrm{~kW}$ water-cooling-type magnetic coupling as the object of study. The coupling's structure is bilaterally symmetric. The left part is taken as the study object and a physical model for it is found, as shown in Figure 4. In the model, the structure used is a copper disk and a steel disk with a linear channel, whose dimensions are indicted in Table 1.

In order to simplify the model, the following assumptions are made [26]: 1) The heat source load is applied to the copper disk's inner part averagely. 2) The heat conductivity coefficient, specific heat, density, and so on, of each component are all constants, which are independent of temperature. 3) The thermal radiation of the coupling's inner electromagnetic wave is ignored. 4) Thermal radiation inside the model is not taken into account. Instead, only heat conductivity and forced convection heat transfer are considered.

Physical models mainly include solid domain parts such as the steel disk, copper disk, permanent magnet disk, and permanent magnet cover. Also included is the fluid domain part consisting of cooling water, among which the rotating speed of the steel disk, copper disk, and cooling water fluid domain is $1500 \mathrm{r} / \mathrm{min}$, and their rotating axis is the $Z$ axis. The greatest attention is paid to the copper disk's temperature distribution and channel's flow field characteristics. Therefore, the channel and copper disk's mesh require a density increase. They are divided by a hexahedral mesh. In addition, the heat transfer coupling surface wall and wall-shadow are split. Fluid-solid coupled thermal calculation is carried out in FLUENT. The physical model and mesh model are shown in Figure 4.

\subsection{Boundary Conditions}

In the simulation, the fluid-solid coupled model and MRF model are adopted [27], different materials are set for different domains, and the rotating domain's speed of

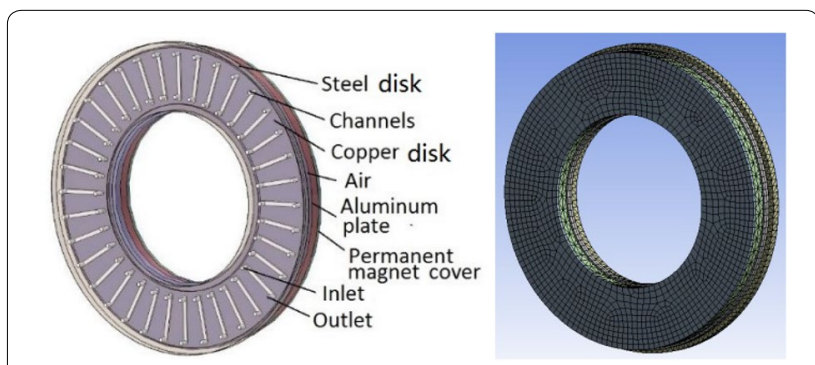

Figure 4 Physical model and mesh model 
Table 1 Size specification of the model

\begin{tabular}{llll}
\hline Name & $\begin{array}{l}\text { Inner diameter/ } \\
\text { length }(\mathbf{m m})\end{array}$ & $\begin{array}{l}\text { Outer diameter/ } \\
\text { width }(\mathbf{m m})\end{array}$ & $\begin{array}{l}\text { Thickness/ } \\
\text { depth } \\
(\mathbf{m m})\end{array}$ \\
\hline Copper disk & 450 & 780 & 10 \\
Steel disk & 420 & 800 & 22 \\
Channel & 120 & 10 & 4 \\
\hline
\end{tabular}

rotation is set to be $1500 \mathrm{r} / \mathrm{min}$. The copper disk will generate heat because of vortex loss. With the focus on the $800 \mathrm{~kW}$ magnetic coupling studied in the present paper, ANSOFT magnetic field simulation is carried out, from which it is clear that the average thermal loss of the copper disk is $1.46 \times 107 \mathrm{~W} / \mathrm{m}^{3}$. The channel inlet is set to be the velocity inlet, and the inlet temperature is $T=313.15$ $\mathrm{K}$. As for the outlet, it is set to be outflow (fully developed, free stream). Under the centrifugal effect, cooling water flows turbulently. The present paper adopts the RNG turbulence flow model, which performs better with rotating flow.

The cooling flow field of the water-cooled permanent magnet coupler is accompanied by the transfer of heat and the heat transfer process satisfies the law of conservation of energy [28]. Specifically, the increase in energy in the fluid element is equal to the net heat flux into the micro-element, and the expression for the sum of the mass force and the surface force on the work performed by the micro-element [29] is as follows:

$$
\begin{array}{r}
\partial(\rho E) / \partial T+\nabla \cdot[\vec{u}(\rho E+P)] \\
=\nabla \cdot\left[k_{e f} \nabla T-\sum_{i} h_{i} J_{i}+\left(\tau_{e f} \cdot \vec{u}\right)\right]+S_{h},
\end{array}
$$

where $E$ is the total energy of the fluid micro-element, $h_{i}$ is the enthalpy of component $i, k_{e f}$ is the effective conduction coefficient, $J_{i}$ is the diffusion flux of component $i, S_{h}$ is the volume heat source. is the eddy current loss heat output of the magnetic field simulation analysis.

The pressure-speed coupled mode employs the SIMPLE calculation method. The pressure difference method adopts the standard pressure difference format, which uses a second-order upwind scheme.

\section{Numerical Simulation Results and Analysis}

\subsection{Simulation Analysis of Different Channel Shapes}

Based on the different shapes of the cooling water channel, many selection schemes for opening channels on the physical model are possible. Considering the heat dissipation effect as well as the processing cost of the channel, the present paper selects the linear flow channel, circular curved channel, and involute curved channel for

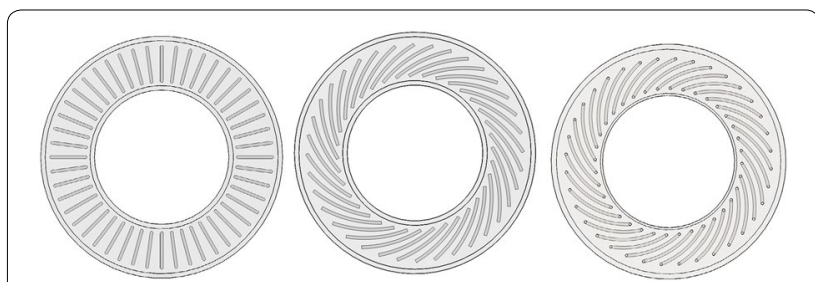

Figure 5 Linear channel, circular curved channel, and involute curved channel

simulation and comparison. Their heat dissipation performance is analyzed. The width of the channel is set as $10 \mathrm{~mm}$, the depth as $4 \mathrm{~mm}$, the central circle diameter of the channel's inlet as $465 \mathrm{~mm}$, the central circle diameter of the channel's outlet as $765 \mathrm{~mm}$, and the number of channels as 36. The model is as shown in Figure 5.

From the FLUENT analog simulation, the results in Table 2 are obtained.

As shown in Table 2, when the thermal loss, $50 \mathrm{~kW}$, of the single-deck copper disk stays the same, the heat release effect under the maximum temperature of the entire body is as follows: the linear channel reduces the temperature by $413{ }^{\circ} \mathrm{C}$, the circular curved channel by $455.4{ }^{\circ} \mathrm{C}$, and the involute curved channel by $474.6{ }^{\circ} \mathrm{C}$. Thus, it is the involute curved channel that achieves the best cooling effect. The details of the maximum temperature of the permanent magnet are as follows: the linear channel reduces the temperature by $135.2^{\circ} \mathrm{C}$, the circular curved channel by $157.4{ }^{\circ} \mathrm{C}$, and the involute curved channel by $167.8{ }^{\circ} \mathrm{C}$. Thus, it is still the involute curved channel that achieves the best cooling effect. The drag torque details are as follows: for clockwise rotation, the drag torque of the linear channel is larger than that of the circular curved channel, whose drag torque exceeds that of the involute curved channel. Therefore, it is the involute curved channel that has the best cooling effect. It reduces the temperature of the entire body's highest temperature rise and the permanent magnet's temperature rise to a great extent with a relatively small drag torque. While designing the coupling, it is necessary to take the direction of rotation into account. In sum, the involute curved channel scheme is the best choice.

The temperature distribution situation can be observed in the input temperature distribution of the different channel types as shown in Figure 6. For the model with the cooling channel, the maximum temperature is in the copper disk, and the minimum temperature is at the inlet of the cooling water. The copper disk's temperature increases gradually from inside to outside along the radial direction. Because of its heat transfer with the copper disk, the cooling water's temperature increases gradually, and the temperature at the parts uncovered by the channel is relatively higher. From the overall temperature 
Table 2 Parameter comparison of temperature results

\begin{tabular}{llcc}
\hline Name & $\begin{array}{l}\text { The maximum temperature of the entire } \\
\text { body }\left({ }^{\circ} \mathrm{C}\right)\end{array}$ & $\begin{array}{l}\text { The maximum temperature } \\
\text { of the permanent magnet }\left({ }^{\circ} \mathrm{C}\right)\end{array}$ & $\begin{array}{l}\text { Drag torque } \\
(\mathbf{N ~ m})\end{array}$ \\
\hline No channel & 544 & 213.6 & 1.26 \\
Linear shape & 131 & 78.4 & 12.23 \\
Circular curved shape & 88.6 & 56.2 & 11.41 \\
Involute curved shape & 69.4 & 45.8 & 10.32 \\
\hline
\end{tabular}

distribution, there is little difference between the temperature distribution of the circular curved channel and that of the involute curved channel. However, the lowtemperature areas in the temperature cloud maps of the two channels are far larger than those of the linear channels. Temperatures of the parts covered by the curved channel are all relatively low. In particular, most of the low-temperature values of the cooling copper disk's involute curved channel are $40-48{ }^{\circ} \mathrm{C}$, while the temperature values of the linear channel copper disk are all above $104{ }^{\circ} \mathrm{C}$. Upon comparison, it is the involute curved channel that has the best cooling effect. Moreover, the temperature distribution at the input end after cooling is also the most reasonable, which brings higher security as well as stability with relatively low processing cost as well as few differences from the circular curved channel. Thus, the involute curved channel is selected to be the cooling channel of the water-cooling-type magnetic coupling.

Figure 7 shows the velocity vector distribution map of the linear channel, circular curved channel, and involute curved channel. From the figure, it can be seen that the directions of flow of the fluid are the same for all three channel types. Cooling water flows out from the outlet after entering from the inlet and getting accelerated through centrifugal force. The maximum velocity of the linear channel is $57 \mathrm{~m} / \mathrm{s}$ and that of the circular curved channel is $64.6 \mathrm{~m} / \mathrm{s}$, the maximum velocity of the involute curved channel is $68.2 \mathrm{~m} / \mathrm{s}$. When the rotating speeds are the same, the involute curved channel achieves the best acceleration effect. Because the curved channel conforms to the rotor's rotating direction and possesses a smoother channel curve, it has a larger acceleration effect and is able to take away more heat, thereby achieving better heat dissipation performance. Further, as the involute channel curve is more adaptable to the direction of rotation, its drag torque value is comparatively smaller, which is more beneficial for the transmission of magnetic torque.

To sum up, among the three types of channels, the cooling effect of the involute channel is the best, which can be observed from the channel type. The ring distribution of the involute curved channel is more average, and the space difference between the cooling water's inlet and outlet is not very large. To reflect the channel distribution situation in a more intuitive way, we have analyzed and studied the influence rule of the specific value $\mathrm{b} / \mathrm{B}$ between the cooling water inlet's minimum space $b$ and the outlet's minimum space B on the cooling effect. As shown in Figure 8, for the linear channel, $\mathrm{b} / \mathrm{B}=32.5 / 57.5=0.565$; for the circular curve channel, $\mathrm{b} / \mathrm{B}=12.8 / 20.2=0.634$; and for the involute curved channel, $\mathrm{b} / \mathrm{B}=15.8 / 16.8=0.94$. The cooling effects of the three show an increasing trend. In other words, the closer the value of $b / B$ is to 1 , the better the cooling effect. When $\mathrm{b} / \mathrm{B}$ is close to 1 , it means that the channel distribution and channel space are average. High-speed revolution imparts a better acceleration effect to the cooling water, which results in a larger cooling effect.

\subsection{Simulation Analysis of Different Number of Channels}

The analog simulation described in the paper showed that the involute curved channel has the best heat dissipation effect, and its drag torque is comparatively smaller. In addition, its processing cost does not rise much compared with that of the circular curved channel. Thus, the involute curved channel is chosen as the object of study, with the objective of analyzing the influence of different number of channel on the model's heat dissipation performance. Although increasing the number of channels can help enhance cooling performance, an excessively large number of channels may lead to serious leakage flux of the copper disk, which would make it unable to transmit the reserved torque. Moreover, too many channels will cause the drag torque to increase. Hence, it is necessary to find the optimal number of channels. The present paper simulates the heat dissipation performance when the thermal losses are the same and the channel numbers are $24,28,32,36,40$, and 44 . The simulation results are shown in Figure 6.

Figure 9(a) plots the impact of the change in the number of channels on the peak temperature rise of the complete machine as well as on the drag torque. From the figure, it can be seen that the peak temperature decreases as the number of channels increases. When there are 36 channels, the decreasing trend slows down. The peak temperature drops from $132{ }^{\circ} \mathrm{C}$ to $99.8{ }^{\circ} \mathrm{C}$, and the drag torque increases as the number of channels increases. 


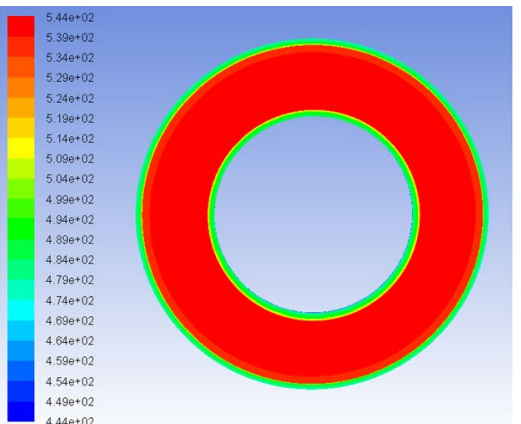

No cooling channel

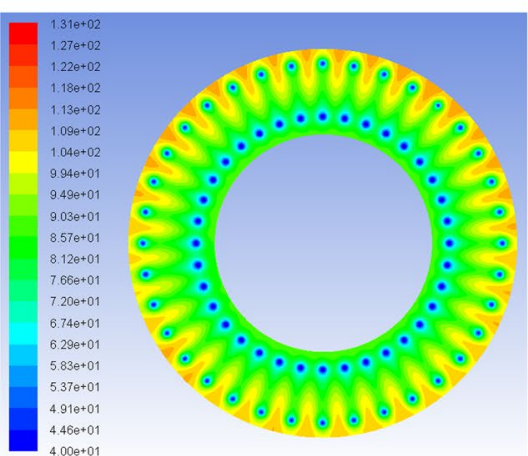

Linear channel

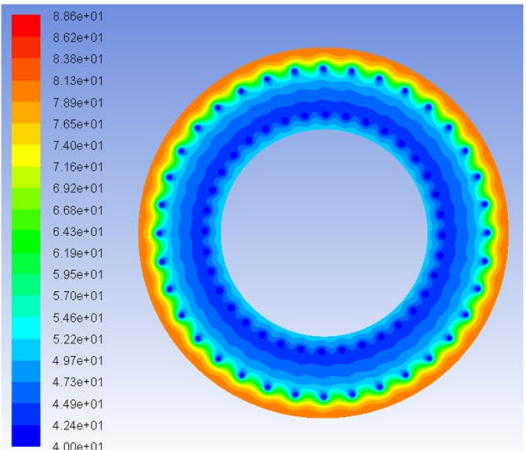

Circular curved channel

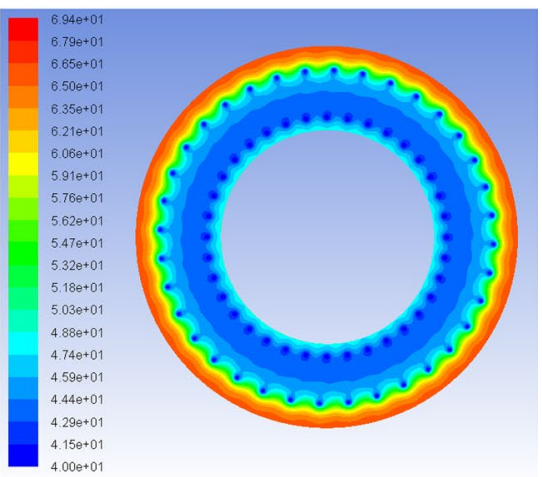

Involute curved channel

Figure 6 Cloud map of temperature distribution at the input end of different channel types $\left({ }^{\circ} \mathrm{C}\right)$
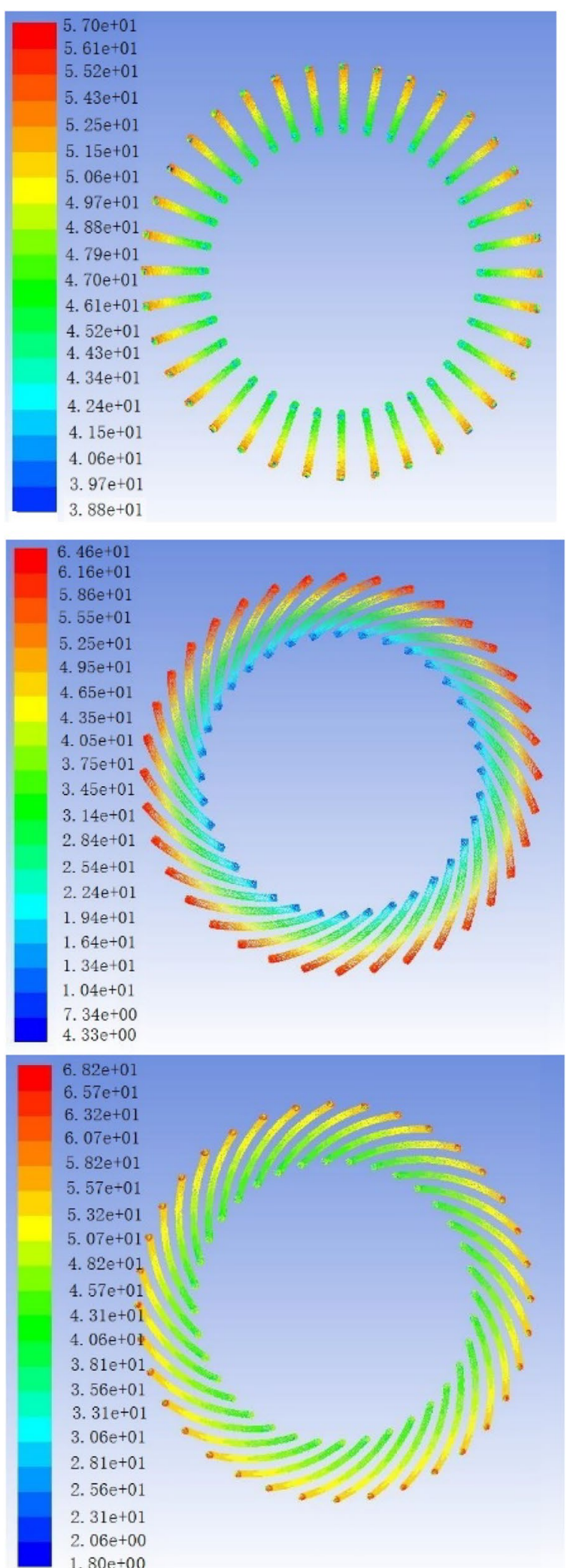

Figure 7 Velocity vector diagram of different channels $(\mathrm{m} / \mathrm{s})$ 


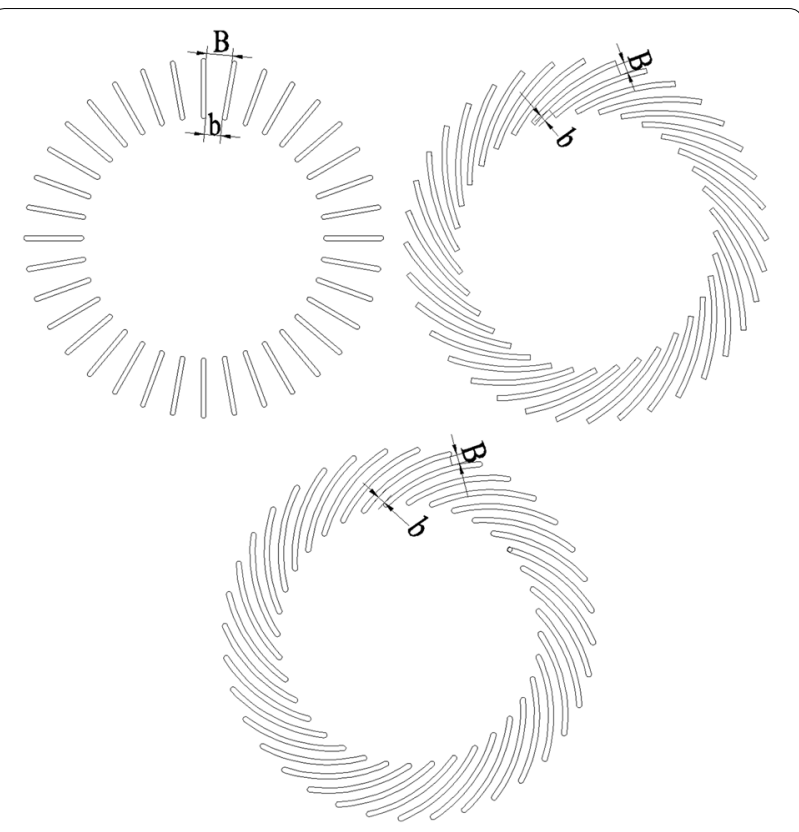

Figure 8 Specific values $b / B$ of inlet and outlet space
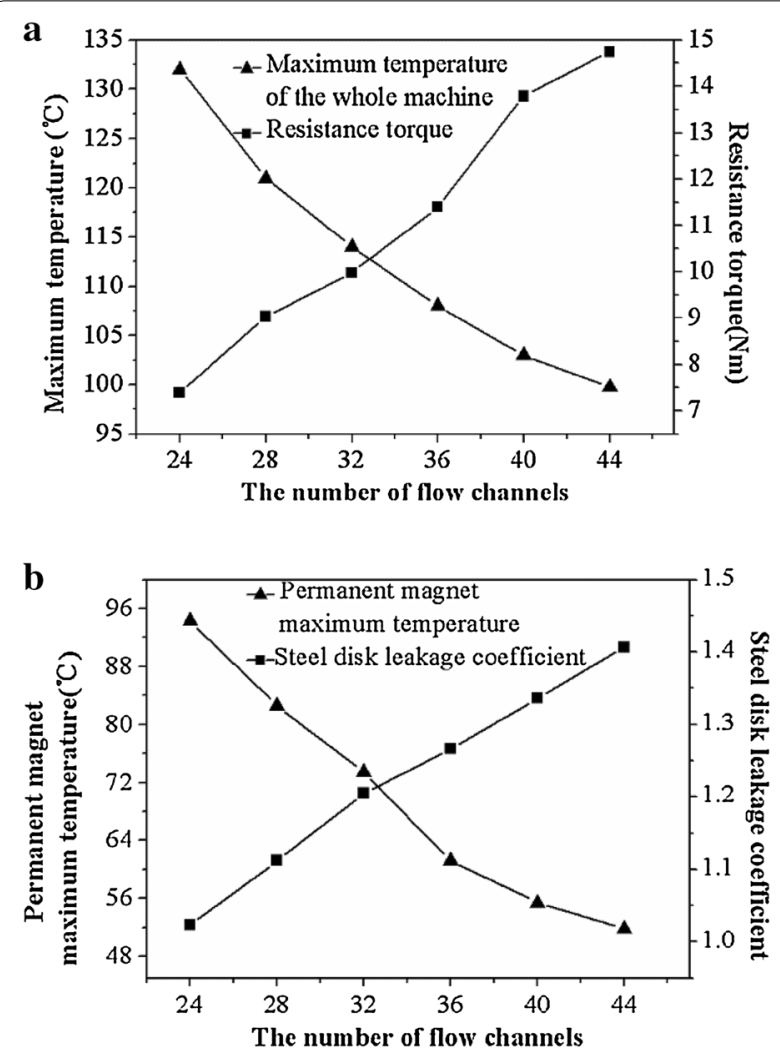

Figure 9 Temperature rise, drag torque, and leakage flux coefficient under different number of channels
When the number of channels changes from 36 to 44, the drag torque rises significantly.

Figure 9(b) plots the impact of the change in the number of channels on the permanent magnet's temperature as well as on the copper disk's leakage flux coefficient. From the figure, it is clear that with the increase in the number of channels, the permanent magnet's peak temperature rise falls. When there are 36 channels, the decreasing tendency slows down. The peak temperature falls from $94.3^{\circ} \mathrm{C}$ to $51.8^{\circ} \mathrm{C}$, and the leakage flux coefficient of the copper disk rises as the number of channels increases, from 1.023 to 1.407 .

Considering that the temperature falls slowly with the increase in the number of channels after 36 channels, and the drag torque as well as the leakage flux increases considerably, the number of cooling channels is selected to be 36 for magnetic coupling, which is relatively reasonable.

\subsection{Simulation Analysis of Different Inlet Velocities}

The number of channels was set to be 36 , and the model was established. Varying inlet velocities were set: 1, 3, $5,7,9,11,13$, and $15 \mathrm{~m} / \mathrm{s}$, respectively, in terms of the boundary conditions. Their influence on the model's heat dissipation performance was analyzed, and the optimal inlet velocity was obtained. The simulation results are shown in Figure 7.

Figure 10(a) plots the impact of the cooling water's different inlet velocities on the complete machine's peak temperature as well as on the drag torque. From the figure, it can be seen that the complete machine's peak temperature rise falls gradually with the increase in the inlet velocity, and the decreasing trend becomes small. After 3 $\mathrm{m} / \mathrm{s}$, the decreasing trend of the peak temperature slows down. However, the complete machine's drag torque increases as the velocity increases. When the flow volume increases, fluid inside the channel increases, causing the drag during rotation to increase; after $3 \mathrm{~m} / \mathrm{s}$, the drag torque's increasing trend is more apparent.

Figure 10(b) plots the impact of the cooling water's different inlet velocities on the permanent magnet's highest temperature. With the increase in the inlet velocity, the permanent magnet's peak temperature rise falls gradually, and the decreasing trend slows down, with $3 \mathrm{~m} / \mathrm{s}$ being the demarcation point. That is to say, when the thermal loss of the magnetic coupling's copper disk is constant and when the cooling water's inlet velocity increases to a certain degree, the heat taken away can cool the copper disk. Therefore, a further increase in velocity will not be advantageous. 

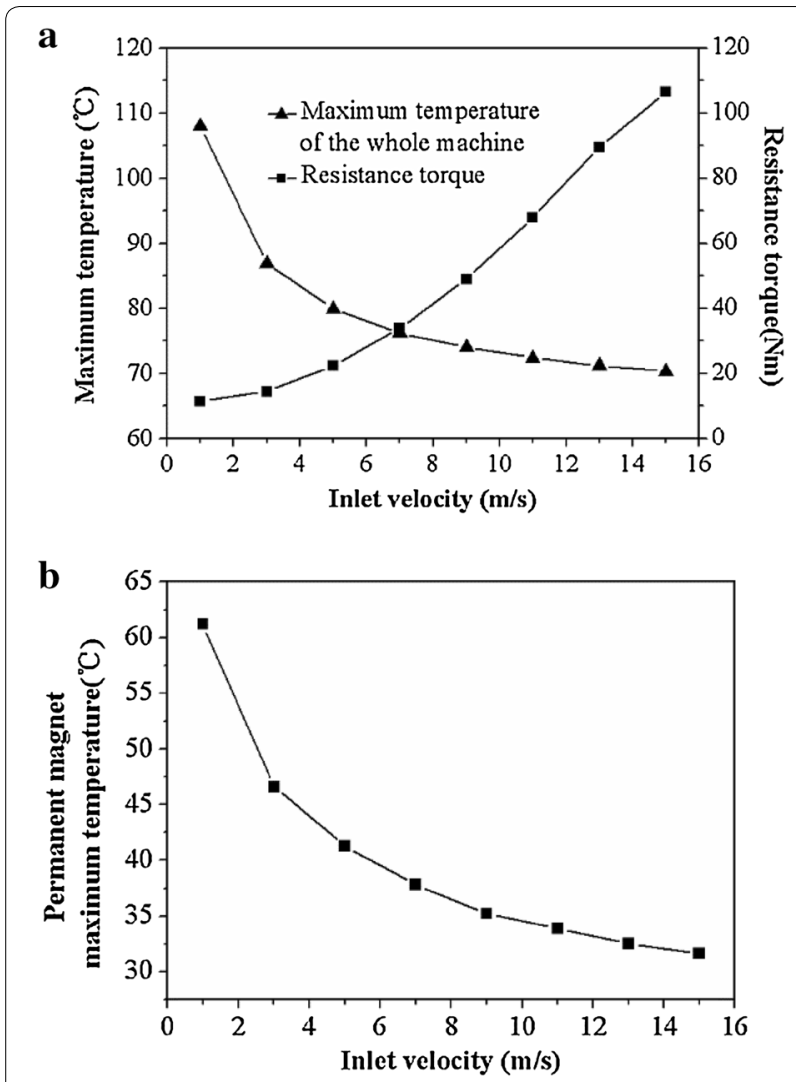

Figure 10 Temperature rise, drag torque under different inlet velocities

The inlet velocity is directly related to the flow volume of the periphery cooling system. Therefore, it is necessary to take the periphery cooling system's circulating water flow and factors, such as the water pump, motor selection, covered area, into comprehensive consideration. It is appropriate to choose $3 \mathrm{~m} / \mathrm{s}$ as the cooling water's inlet velocity.

\section{Experimental Verification}

\subsection{Experimental Platform}

In order to verify the accuracy of the method that uses fluid-solid coupled heat transfer and the MRF model to calculate the rotating cooling temperature field, which is proposed in the present paper, and also to study the distribution of the water cooling coupling temperature field during the actual operational process, in order to optimize the coupling's structure, an experimental platform is established for verification and analysis.

The experimental platform is composed of an $800 \mathrm{~kW}$ water-cooling magnetic coupling, an infrared thermometer sensor, a torque speed sensor, an $800 \mathrm{~kW}$ mineused anti-explosion drive motor, an $800 \mathrm{~kW}$ mine-used

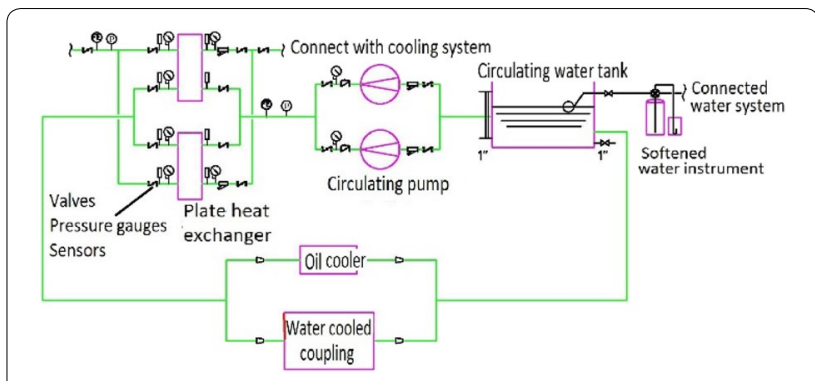

Figure 11 Water cooling coupling experimental schematic

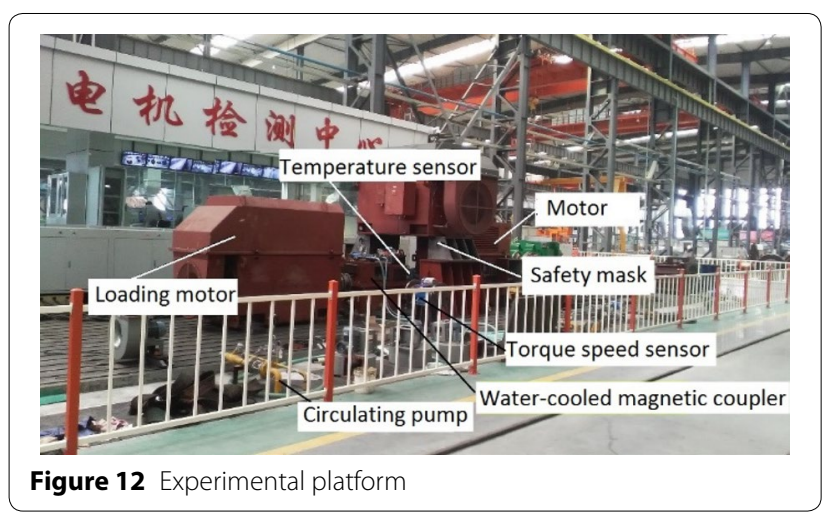

anti-explosion loading motor, a control system, and a water cooling circulating system. Among them, the water-cooling magnetic coupling's cooling end employs the involute curved channel. The number of channels is 36. The diameters of the cooling water's inlet and outlet are each $10 \mathrm{~mm}$, and the thickness of the channel is $4 \mathrm{~mm}$. The water cooling circulating system includes the periphery oil and water heat exchanger, inlet and outlet piezometer, thermometer, electromagnetic flowmeter, circulating water pump, and water tank. The experimental process is shown in Figure 11, and the experimental platform is shown in Figure 12.

The operating principle of the experimental platform is as follows: the power is turned on, and the drive motor starts rotating, driving the input end's rotor to rotate. At this time, relative movement occurs inside the coupling, generating a magnetic field. Under the effect of the vortex magnetic field, the loading motor starts rotating. The electric actuator is adjusted to change the gap between the permanent magnet and the copper disk, in order to change the output power. The PC machine and controller manipulate the drive motor and load. A torque speed sensor is installed under the velocity-measuring fluted disk of the input and output ends, measuring the input and output rotating speeds; a thermometer is installed at the pipe section of the coupling's cooling water inlet 


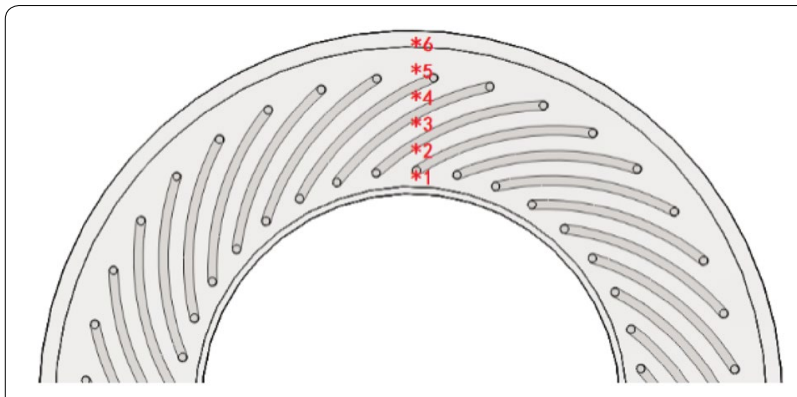

Figure 13 Measuring points of temperature sensor

and outlet, measuring the temperature of the inlet and outlet water; an electromagnetic flowmeter is installed at the outlet of the pump, measuring the flow volume of the cooling water; and a temperature sensor is installed inside the box, measuring the temperatures of different parts of the copper disk. From the water temperatures of the cooling water inlet and outlet, the heat dissipation capacity of the cooling water can be calculated. Moreover, the copper disk's temperature measured by the infrared thermometer sensor and the simulated value are compared.

The experimental scheme is described in the following: (1) To verify the correctness of the temperature field of the involute curved channel calculated with the fluidsolid coupled heat transmission and MRF model, six uniformly spaced temperatures are utilized for verification. In other words, a temperature sensor is used to collect stable values of the six measuring points as shown in Figure 13, and they are compared with the simulation calculation results from a single copper disk of $50 \mathrm{~kW}$ and $70 \mathrm{~kW}$. (2) The variation in the temperature data up to stability are collected at each point. The characteristics of the temperature are analyzed so as to provide reference points for further optimization of the channel.

\subsection{Experimental Process and Results}

\subsubsection{Verification of Simulation Results}

The experimental process consists of the following steps: the electric actuator is adjusted to maximize the air gap of the coupling $(34 \mathrm{~mm})$. Then the drive motor is started, driving the magnetic coupling's outer cover to rotate at the nominal rotating speed of $1480 \mathrm{r} / \mathrm{min}$; the loading motor is static. The aperture of the actuator is then used to gradually decrease the coupling's air gap. The transmission torque increases gradually. The loading motor will start during the process of reducing the air gap, until its rotating speed reaches $1315 \mathrm{r} / \mathrm{min}$. At this time, the slip ratio is approximately $12.5 \%$, and the single disk's thermal loss is $50 \mathrm{~kW}$. The motor is kept running for sufficient time until the collecting temperature displayed on the controller does not change. The experiment is now halted. The coupling is left alone until its temperature reaches the indoor temperature. The above process is repeated until the loading motor's rotating speed is $1260 \mathrm{r} / \mathrm{min}$, the slip ratio is $15 \%$, and the single disk's thermal loss is $70 \mathrm{~kW}$. The motor is kept running for sufficient time until the collecting temperature displayed on the controller does not change. The experiment is then halted.

The comparison between the experimental temperature and the simulation temperature at the six measuring points is as shown in Figure 14. Under the two situations where the single disk's thermal losses are $50 \mathrm{~kW}$ and 70 $\mathrm{kW}$, the variations in the simulation value and experimental value of the temperature are consistent: the peak temperatures are both at position 6 , and the minimum temperatures are both at position 1. There is no cooling channel at position 6 , and therefore the temperature rise is obvious; position 1 corresponds to the inlet of the cooling water and contacts low-temperature cooling water. Therefore, the cooling effect is the best, and the
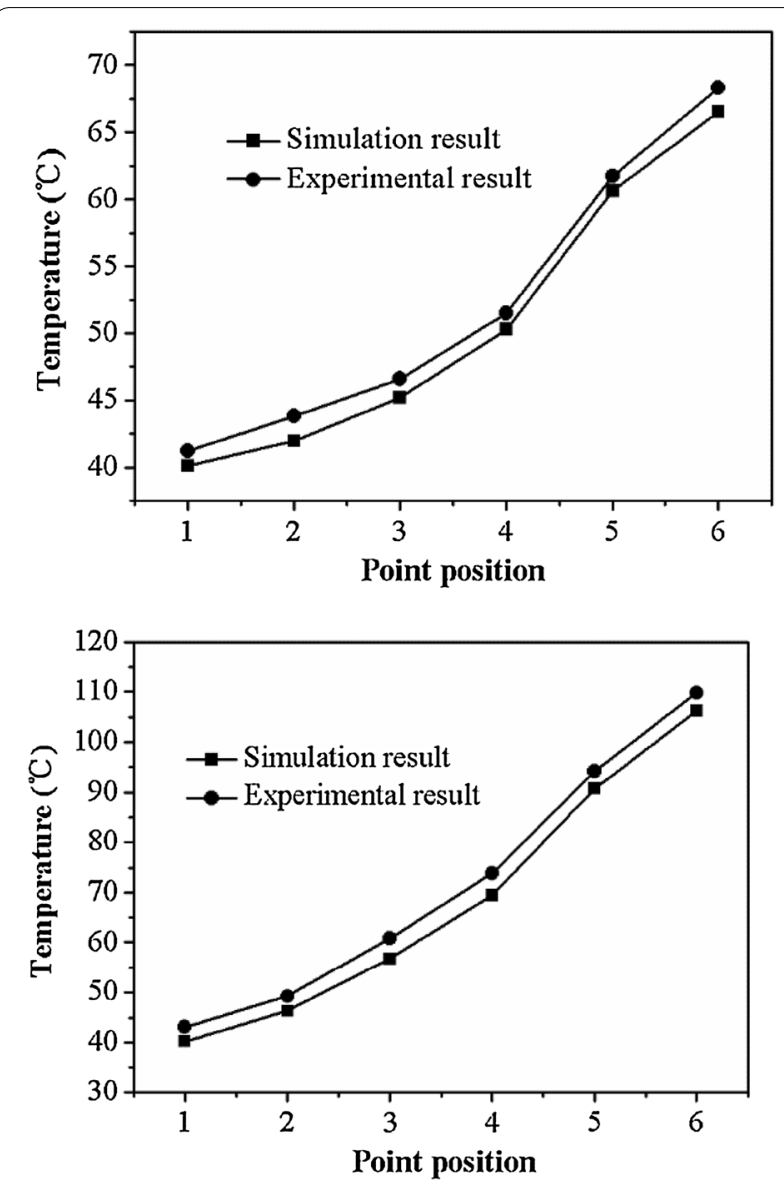

Figure 14 Comparison between the experiment value and temperature value at the measuring points 


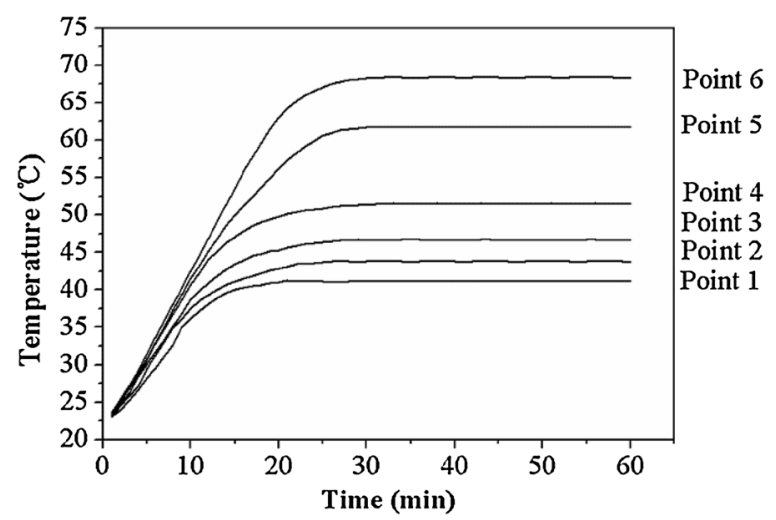

Figure 15 Changing curve of output temperature when copper disk's thermal loss is $50 \mathrm{~kW}$

temperature is the lowest. The temperature differences between the simulation value and experimental value are both within $4{ }^{\circ} \mathrm{C}$. The error is within the permissible range, proving the correctness of the method of fluidsolid coupled heat transmission combined with the MRF model.

\subsubsection{Analysis of the Change in Temperature Field}

The values at each point are collected when the slip ratio is $12.5 \%$ and the single disk's thermal loss is $50 \mathrm{~kW}$ in order to analyze the variation, as shown in Figure 15. The experiment starts at 09:09:12, when the temperature is the indoor temperature, namely, $23^{\circ} \mathrm{C}$. With the rotation of the motor, temperatures at all the points start rising and then begin to stabilize. Moreover, the higher the stable temperature value, the longer the time to reach a stable value; this is explained by the fact that the initial slip of the coupling is relatively large and thus produces relatively more heat. However, the copper rotor's rotating speed is not high, the cooling water's flow speed is low, and the heat dissipation capacity is poor. As a result, the temperatures gradually rise; when the slip becomes constant, the heat production volume is fixed, and the cooling water's flow speed becomes the same as the copper rotor's rotating speed" can be used, the heat convection coefficient is constant, and the temperature finally stabilizes. The higher the stable temperature value, the longer the time needed to reach a stable value when the heat dissipation capacity is fixed.

Comparison between the experimental value and the simulation value shows that the experimental result is higher than the simulation result. The following analysis explains this error: 1) The flow speed of the cooling water used in the simulation calculation is constant, and the distribution inside the channel is average. Nevertheless, in the experiment, the cooling water will be biased toward the channel under the centrifugal effect of rotation, and will be unable to fill the channel completely. Therefore, the cooling effect is not as good as the simulation result. 2) During the experimental process, the bearing will produce a temperature rise while rotating at a high speed, which will be transmitted to the copper disk through heat conduction and thus increase the copper disk's temperature. However, the heat is not considered in the analog simulation. That is, the heat used in the simulation is lower than the actual heat in the experiment. 3) In the experiment, the temperature of the peripheral cooling water heat transfer system will rise to some extent after a long period of heat exchange. The cooling water's temperature increases after heat transfer, when the effect of heat transfer decreases. However, in the simulation calculation, the inlet temperature of the cooling water is constant, and the heat transfer effect is constant. Thus, its heat dissipation effect is better.

In a practical application of high-power water cooling magnetic coupling, only the inlet and outlet water temperatures of the circulating cooling water can be measured. It is impossible to know the temperatures of vulnerable key parts, such as the copper disk and permanent magnet accurately. At present, the solution method proposed in the present paper can be used for calculation in order to predict the temperatures of the copper disk and permanent magnet. In this way, when the temperature becomes excessively high, protective measures can be taken to safeguard the copper disk and permanent magnet to prevent malfunctions caused by high temperature rise, such as softening of the copper disk and demagnetizing of the permanent magnet. Therefore, the solution put forward in the paper has high practical application value.

\section{Conclusions}

To validate the simulation method of the fluid-solid heat transfer model combined with the MRF model proposed in the paper, a simulation calculation is carried out for the high-speed rotating water-cooling-type magnetic coupling's temperature field, which is relatively less studied. The cooling channel is optimized and the proposed simulation method verified through experiment. The following conclusions are drawn:

(1) Using the finite element method, a temperature field simulation calculation is carried out for four different channel types: no channel, linear channel, circular curve channel, and involute curved channel. The results show that the heat dissipation performance and torque transmission of the involute curved channel are the best. Therefore, the involute curved channel is the right choice. 
(2) Different numbers of channels are investigated. The results show that when there are 36 channels, the heat dissipation effect can meet the requirements. At this time, the increase in the number of channels will not enhance the cooling effect much, yet the drag torque will clearly increase. Thus, the number of channel numbers is set to be 36 .

(3) Different inlet velocities of cooling water are studied. It is discovered that the heat dissipation effect is ideal when the velocity is $3 \mathrm{~m} / \mathrm{s}$. At this time, the increase in velocity has little influence on the heat dissipation effect, but the drag torque will rise significantly, and the requirements for the periphery cooling system become stricter. Thus, $3 \mathrm{~m} / \mathrm{s}$ can be set as the optimal inlet velocity.

(4) The experiment is used to verify the water-coolingtype magnetic coupling's temperature field with 36 involute curved channels, an inlet velocity of $3 \mathrm{~m} / \mathrm{s}$, and heat sources of $50 \mathrm{~kW}$ and $70 \mathrm{~kW}$. The results are close to the simulation calculation results, which proves the correctness of the proposed method. Meanwhile, a study has been conducted on the variation in the actual temperature field, in order to provide reference points for the optimization of the structure.

(5) Although it is impossible to measure the real-time temperature of the vital components inside the coupling, such as the copper disk and permanent magnet, the method proposed in the paper can be used to calculate and predict the temperature distribution, in order to prevent component malfunctions caused by an excessive temperature rise.

\section{Authors' Contributions}

LW was in charge of the whole trial; YZ was responsible for numerical simulation and wrote the manuscript. All authors read and approved the final manuscript.

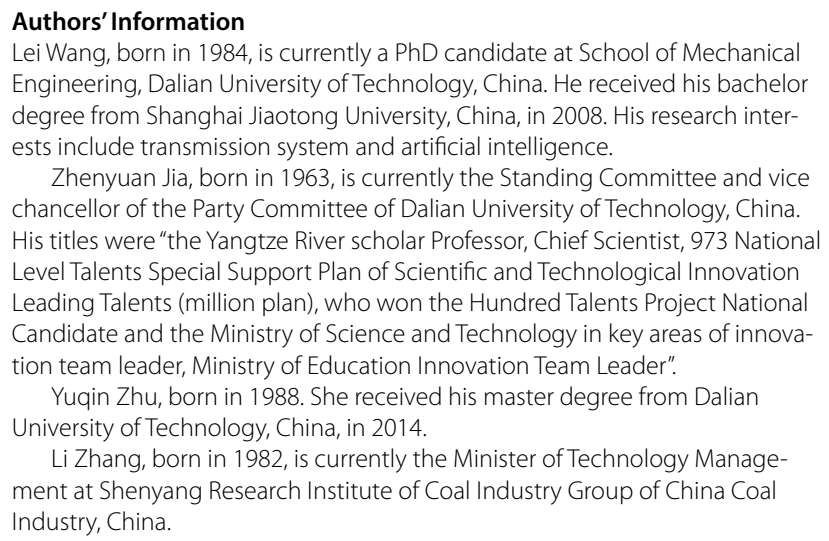

Zhenyuan Jia, born in 1963, is currently the Standing Committee and vice chancellor of the Party Committee of Dalian University of Technology, China. His titles were "the Yangtze River scholar Professor, Chief Scientist, 973 National Level Talents Special Support Plan of Scientific and Technological Innovation Leading Talents (million plan), who won the Hundred Talents Project National Candidate and the Ministry of Science and Technology in key areas of innovation team leader, Ministry of Education Innovation Team Leader".

Yuqin Zhu, born in 1988. She received his master degree from Dalian University of Technology, China, in 2014.

Li Zhang, born in 1982, is currently the Minister of Technology Management at Shenyang Research Institute of Coal Industry Group of China Coal Industry, China.

\section{Acknowledgements}

The authors sincerely thanks to Professor Zhenyuan Jia of Dalian University of Technology for his critical discussion and reading during manuscript preparation.

\section{Competing Interests}

The authors declare that they have no competing interests.

\section{Funding}

Supported by China Coal Science and Technology Group Technology Innovation Fund Major Project (Grand No. 2018ZD002), and China Coal Science and Technology Group Technology Innovation Fund Youth Project (Grand No. 2018-2-QN010).

\section{Author Details}

${ }^{1}$ School of Mechanical Engineering, Dalian University of Technology, Dalian 116023, China. ${ }^{2}$ State Key Laboratory of Coal Mine Safety Technology, CCTEG Shenyang Research Institute, Fushun 113122, China.

Received: 23 March 2018 Revised: 21 February 2019 Accepted: 24 June 2019

Published online: 04 July 2019

\section{References}

[1] S Mohammadi, M Mirsalim. Design optimization of double-sided permanent-magnet radial-flux eddy-current couplers. Electric Power Systems Research, 2014, 108(3): 282-292.

[2] B Y Zhang, Y A Wan, Y Li, et al. Optimized design research on adjustablespeed permanent magnet coupling. IEEE International Conference on Industrial Technology, 2013: 380-385.

[3] Z H Guo, HW Hou, R L Feng, et al. Application of permanent magnetic coupling to soft starting in mining machinery. Colliery Mechanical \& Electrical Technology, 2016(5): 91-93.

[4] Y L Jiang. Study on magnetic coupling's temperature field and dynamic performance. Dalian Jiaotong University, 2015. (in Chinese)

[5] G Cheng, Y C Guo, SWang, et al. Analysis on magnetic coupling's heat dissipation disk's heat transmission performance and improvement of structural parameters. CA Database, 2017, 14(9): 22-27+ 3-4. (in Chinese)

[6] Huifang Liu, Xingwei Sun, Yifei Gao, et al. Magnetostrictive and kinematic model considering the dynamic hysteresis and energy loss for GMA. Chinese Journal of Mechanical Engineering, 2017, 30(2): 241-255.

[7] H K Chen, B F Zhang, L P Zhu. Finite element analysis of temperature field on permanent magnetic coupler. Colliery Mechanical \& Electrical Technology, 2017(5): 46-49. (in Chinese)

[8] MY Dai, Z S Sun. Numerical simulation and experimental study on magneto-thermal bidirectional coupling heat transfer of permanent magnet governor. Mechatronics, 2017(06): 18-23. (in Chinese)

[9] Z S Sun, X Q Li, X N Li. Research and application of permanent magnet eddy current governor. Machinery Manufacturing \& Automation, 2016, 45(3): 1-4. (in Chinese)

[10] D Zheng, D ZWang, TY Shi, et al. Design optimization of permanent magnet couplings. Control \& Decision Conference, IEEE, 2015: http://r.cnki.net/ KCMS/detail/detail.aspx?dbcode $=$ MTZI\&dbname $=$ MTZILAST2015\&filen ame=KZJC201505001016\&.

[11] JW Shi, Z L Wang, K Sun, et al. Analysis of eddy current loss and temperature field of permanent magnetic coupling for mine. Safety in Coal Mines, 2017, 48(10): 101-104+107. (in Chinese)

[12] SY Tan, Z A Zhang. Design on intelligent controller of magnetic controlled soft starting for reactive compensation. International Conference on Computational Intelligence and Industrial Application, 2010: http://r.cnki. net/KCMS/detail/detail.aspx?dbcode=MTZI\&dbname=MTZI8115\&filen ame $=$ ZNXX201012026092\&.

[13] J K Cai, Y B Cai, X F Zhang. Simulation study on a new type inlet hole of permanent magnet magnetic coupling. Journal of Mechanical Transmission, 2017, 41 (06): 169-173. (in Chinese)

[14] PX Lian. Research on heating and heat dissipation of permanent magnet synchronous in-wheel motor. Harbin Institute of Technology, 2013. (in Chinese) 
[15] H Q Li. Study on water cooled heat dissipation characteristics of motor controller of pure electric vehicle. Hefei University of Technology, 2016. (in Chinese)

[16] YXYin, ZX Zhu. Research of water cooling performance of mine flameproof frequency convector. Coal Mine Machinery, 2015, 36(8): 83-85. (in Chinese)

[17] YX Chen. Fluid field calculation and cooling system design for $45 \mathrm{kw}$ water-cooling vehicle moto. Harbin University of Technology, 2013. (in Chinese)

[18] Nipun Thamatam, J John, Sandilya Sridhara. A study on offset drift with temperature of MEMS inertial sensors. International Conference on Smart Sensors \& Systems. IEEE, 2017: https://ieeexplore.ieee.org/docum ent/7873604/.

[19] Y Wang, Y Hu, Q Huang, et al. Transient heat transfer study of direct contact condensation of steam in spray cooling water. Transactions of Tianjin University, 2017: http://link.springer.com/10.1007/s12209-017-0106-6.

[20] M M Sarafraz, A Arya, F Hormozi, et al. On the convective thermal performance of a CPU cooler working with liquid gallium and CuO/water nanofluid: A Comparative study. Applied Thermal Engineering, 2017, 112: 1373-1381.

[21] Z P Chen. Analysis and research on axial force and temperature field of variable speed asynchronous magnetic coupling. Jiangsu University, 2014 (in Chinese)

[22] X H Hao, B Peng, Y Chen, et al. Transient thermal model of a permanent magnet synchronous planar motor considering spreading thermal resistance. Applied Thermal Engineering, 2015, 81(1): 1-9.
[23] W Ou, M Yang, F Meng, et al. Continuous high-performance drive of rotary traveling-wave ultrasonic motor with water cooling. Sensors \& Actuators A Physical, 2015, 222: 220-227.

[24] H J Song, Q Yan, X D Zhu, et al. Design and research on the cooling effect of a computer water cooling system. Research and Exploration in Laboratory, 2017, 36(03): 55-58.

[25] M Y Dai. Multi-field coupling study on high-power water-cooling permanent magnetic vortex governor. Nanjing University of Science and Technology, 2017. (in Chinese)

[26] M A Mahmud, B D Macdonald. Experimental investigation of interfacial energy transport in an evaporating sessile droplet for evaporative cooling applications. Physical Review E, 2017, 95(1): 012609.

[27] Y Sun, Z Guan, H Gurgenci, et al. A study on multi-nozzle arrangement for spray cooling system in natural draft dry cooling tower. Applied Thermal Engineering, 2017, 124: 795-814.

[28] Y Kamla, M Bouzit, H Ameur, et al. Effect of the inclination of baffles on the power consumption and fluid flows in a vessel stirred by a Rushton turbine. Chinese Journal of Mechanical Engineering, 2017, 30(4): 1008-1016.

[29] D S Arnold, A Tura, A Ruebsaat-Trott, et al. Design improvements of a permanent magnet active magnetic refrigerator. International Journal of Refrigeration, 2014, 37(1): 99-105.

\section{Submit your manuscript to a SpringerOpen ${ }^{\circ}$ journal and benefit from:}

- Convenient online submission

- Rigorous peer review

- Open access: articles freely available online

- High visibility within the field

- Retaining the copyright to your article

Submit your next manuscript at $\boldsymbol{\nabla}$ springeropen.com 